\title{
Brain and Lung Imaging Correlation in Patients with COVID-19: Could the Severity of Lung Disease Reflect the Prevalence of Acute Abnormalities on Neuroimaging? A Global Multicenter Observational Study
}

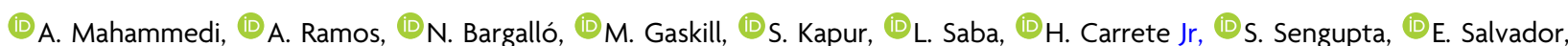

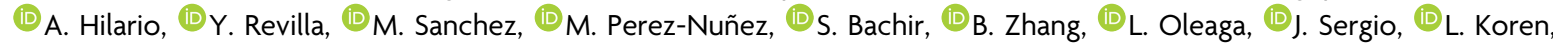

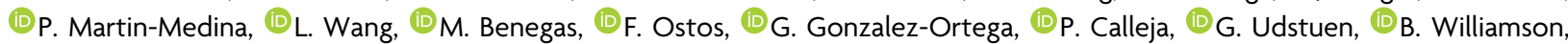

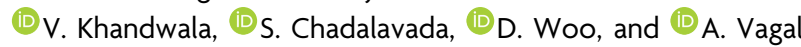

\begin{abstract}
PURPOSE: Our aim was to study the association between abnormal findings on chest and brain imaging in patients with coronavirus disease 2019 (COVID-19) and neurologic symptoms.

MATERIALS AND METHODS: In this retrospective, international multicenter study, we reviewed the electronic medical records and imaging of hospitalized patients with COVID-19 from March 3, 2020, to June 25, 2020. Our inclusion criteria were patients diagnosed with Severe Acute Respiratory Syndrome coronavirus 2 (SARS-CoV-2) infection with acute neurologic manifestations and available chest $\mathrm{CT}$ and brain imaging. The 5 lobes of the lungs were individually scored on a scale of $0-5$ ( 0 corresponded to no involvement and 5 corresponded to $>75 \%$ involvement). A CT lung severity score was determined as the sum of lung involvement, ranging from 0 (no involvement) to 25 (maximum involvement).

RESULTS: A total of 135 patients met the inclusion criteria with 132 brain CT, 36 brain MR imaging, 7 MRA of the head and neck, and 135 chest CT studies. Compared with 86 (64\%) patients without acute abnormal findings on neuroimaging, 49 (36\%) patients with these findings had a significantly higher mean $C T$ lung severity score (9.9 versus 5.8, $P<.001)$. These patients were more likely to present with ischemic stroke (40 [82\%] versus $11[13 \%], P<.0001)$ and were more likely to have either ground-glass opacities or consolidation (46 [94\%] versus $73[84 \%], P=.01$ ) in the lungs. A threshold of the $C T$ lung severity score of $>8$ was found to be $74 \%$ sensitive and $65 \%$ specific for acute abnormal findings on neuroimaging. The neuroimaging hallmarks of these patients were acute ischemic infarct $(28 \%)$, intracranial hemorrhage (10\%) including microhemorrhages (19\%), and leukoencephalopathy with and/or without restricted diffusion (11\%). The predominant CT chest findings were peripheral ground-glass opacities with or without consolidation.
\end{abstract}

CONCLUSIONS: The CT lung disease severity score may be predictive of acute abnormalities on neuroimaging in patients with COVID19 with neurologic manifestations. This can be used as a predictive tool in patient management to improve clinical outcome.

ABBREVIATIONS: COVID-19 = coronavirus disease 2019; GGOs = ground-glass opacities; PRES = posterior reversible encephalopathy syndrome; SARSCoV-2 $=$ Severe Acute Respiratory Syndrome coronavirus 2; TIPIC $=$ Transient Perivascular Inflammation of the Carotid artery syndrome

$\mathbf{S}$ evere Acute Respiratory Syndrome coronavirus 2 (SARSCoV-2) began in Wuhan, China, in December 2019 and has rapidly spread around the world to become a pandemic. ${ }^{1}$

Received October 26, 2020; accepted after revision January 4, 2021

From the Departments of Neuroradiology, (A.M., A.V., M.G., L.W., G.U., B.W., V.K.), Cardiopulmonary Imaging, (S.K.), Neurology (S.S., D.W.), and Department of Informatics and Interventional Radiology, (S.C.), University of Cincinnati Medical Center, Cincinnati, Ohio; Departments of Epidemiology and Biostatistics (B.Z.) and Pediatrics, (S.B.), Cincinnati Children's Hospital Medical Center, Cincinnati, Ohio; Departments of Neuroradiology (A.R., E.S., A.H., L.K., P.M.-M.), Cardiopulmonary Imaging (Y.R., M.P.-N.) and Neurology (F.O., G.G.-O., P.C.), Hospital Universitario 12 de Octubre, Madrid, Spain; Departments of Neuroradiology (L.O., N.B.) and Cardiopulmonary Imaging (M.S., M.B., J.S.), Hospital Clínic de Barcelona, Sunyer Biomedical Research Institute, Barcelona, Spain; Department of Neuroradiology
Extensive studies have described chest and brain imaging characteristics associated with coronavirus disease 2019 (COVID19). ${ }^{2-13}$ The hallmarks of COVID-19 infection on chest imaging

(L.S.), Azienda Ospedaliero Universitaria di Cagliari, Monserrato (Cagliari), Italy; Department of Neuroradiology (H.C.), Escola Paulista de Medicina, Federal University of São Paulo, São Paulo, Brazil.

Please address correspondence to Abdelkader Mahammedi, MD, Department of Radiology, University of Cincinnati Medical Center, 234 Goodman St, Cincinnati, OH 45267-0525; e-mail: abdelkader.mahammedi@uc.edu

- Indicates open access to non-subscribers at www.ajnr.org

EIndicates article with online supplemental data.

http://dx.doi.org/10.3174/ajnr.A7072 
are now well-established, including bilateral and peripheral ground-glass and consolidative pulmonary opacities. ${ }^{2-5}$ COVID19-related brain imaging findings such as ischemic infarcts, hemorrhages, and multiple patterns of leukoencephalopathy ${ }^{6-13}$ are also well-known. The clinical symptomatology has been linked to the imaging findings with up to $47 \%$ of patients with COVID-19 with neurologic symptoms demonstrating acute neuroimaging findings ${ }^{6}$ and patients with high lung severity scores being admitted to the intensive care unit. ${ }^{3}$ The incidence of neurologic symptoms is higher in patients with more severe respiratory disease. ${ }^{10,13}$ There is increasing evidence that patients with acute lung injury are at risk of brain injury through hypoxemia and/or proinflammatory mediators that connect both the brain and the lungs. ${ }^{14-17}$ However, little information is available on the potential association between the prevalence of neuroimaging abnormalities and the severity of CT lung findings in patients with COVID-19. The objective of this study was to examine the association between chest and brain imaging abnormalities in patients with COVID-19. We hypothesized that the severity of lung disease may predict acute abnormalities on neuroimaging in patients with COVID-19 with neurologic symptoms.

\section{MATERIALS AND METHODS \\ Study Design and Patient Population}

We used a retrospective, multicenter study design from 5 academic institutions in 4 countries: Spain, Italy, Brazil, and the United States (University of Cincinnati Medical Center, Cincinnati, Ohio; University Hospital 12 de Octubre, Madrid, Spain; Hospital Clínic de Barcelona, Barcelona, Spain; University of Cagliari, Cagliari, Italy; and Federal University of Sao Paulo, Sao Paulo, Brazil). Institutional review board approval and waivers were obtained at all institutions. Our inclusion criteria were the following: 1) hospitalized patients who were positive for COVID-19 via real-time polymerase chain reaction of respiratory secretions obtained by bronchoalveolar lavage, endotracheal aspirate, nasopharyngeal swab, or oropharyngeal swab from March 3 to June 25, 2020; 2) the presence of acute neurologic symptoms during the hospital stay; 3) available chest CT and brain CT and/or MR imaging. We reviewed the electronic medical records to extract clinical, laboratory, and demographic data.

\section{Image Acquisition}

All noncontrast brain and chest CT scans were obtained as per standard-of-care protocols. The MR imaging of the brain and MRA of the head and neck were performed on 1.5T scanners with standardized protocols. Gd-DTPA $(0.1 \mathrm{mmol} / \mathrm{kg}$ of gadobutrol, Gadovist; Bayer Schering Pharma) was used for contrast studies.

\section{Image Interpretation}

For each patient, all neuroimaging was evaluated for the following characteristics: 1) T2/FLAIR white matter signal abnormality: nonconfluent punctate and/or confluent white matter lesions with and/or without restricted diffusion and/or microhemorrhage; 2) enhancement: leptomeningeal, parenchymal, and cranial nerves; 3) acute ischemic infarcts: vascular territory, small/ watershed zone, and cardioembolic; 4) intracranial hemorrhages: parenchymal, subarachnoid, and microhemorrhage; 5) acute leukoencephalopathy; 6) posterior reversible encephalopathy syndrome (PRES); and 7) hypoxic-ischemic encephalopathy. All neuroimaging was divided into 2 groups: acute-versus-nonacute neuroimaging findings. Patients with chronic microvascular ischemic disease, remote infarcts, or chronic demyelinating disease but without acute neuroimaging findings were counted in the group of nonacute neuroimaging. Scans were compared with prior examinations if available. Imaging findings without prior neuroimaging were considered acute if compatible with acute patient symptoms. The mean timeframe difference between the scans of patients with acute-versus-nonacute neuroimaging was 2 days.

Chest CT scans were evaluated for the following characteristics: 1) the presence and/or absence of ground-glass opacities (GGOs) and/or consolidation; 2) crazy paving pattern; 3) cavitation, pleural effusions, or lymphadenopathy; 4) CT lung severity score; 5) predominant zonal distribution; and 6) lobar involvement. A semiquantitative CT-based severity score of pulmonary disease burden was measured by counting the involvement of lung lobes similar to that in Pan et al. ${ }^{4}$ Each of the 5 lung lobes was scored from 0 to 5 as the following: 0 , no involvement; one, $<5 \%$ involvement; two, 25\% involvement; three, $26 \%-49 \%$ involvement; four, $50 \%-75 \%$ involvement; and five, $>75 \%$ involvement. The CT lung severity score was the sum of the individual lobar scores and ranged from 0 (no involvement) to 25 (maximum involvement). In patients with multiple chest CT scans, only scans with the greatest severity were selected, and all were performed approximately 7-12 days after the initial onset of respiratory symptoms. All scans were reviewed by 6 neuroradiologists and 5 chest radiologists in consensus.

\section{Statistical Analysis}

Analyses were performed to describe the distribution of the variables of interest. Continuous variables were presented as mean [SD] or median and range. The normality of continuous variables was assessed by the Kolmogorov-Smirnov test. Categoric variables were presented as frequencies and percentages. Continuous variables were compared using the 2-sample $t$ test or Wilcoxon rank sum test. Proportions for categoric variables were compared using the $\chi^{2}$ test or Fisher exact test. The logistic regression model was used to assess the association between abnormalities on acute neuroimaging and the CT lung severity score. Prediction ability was evaluated using a receiver operating characteristic curve. The optimal threshold and the sensitivity and specificity at the threshold were reported. All statistical analyses were performed using STATA software (Version 15; StataCorp). The significance threshold was set at a 2 -sided $P$ value $<.05$.

\section{RESULTS}

A total of 172 consecutive hospitalized patients with COVID-19 with acute neurologic symptoms were reviewed. Of these, 135 patients fit our inclusion criteria (Fig 1, flow chart). The demographic and neurologic clinical characteristics are shown in the Online Supplemental Data. The mean age was 68.2 [SD, 15.1 years (age range, 17-94 years), and 86 (64\%) were men. 
172 consecutive hospitalized COVID-19 patients with acute neurological symptoms and referred for neuroimaging from March 3 to June 25, 2020

135 patients had chest $\mathrm{CT}$, developed acute neurological symptoms and received neuroimaging

- $132(98 \%)$ received noncontract head CT

- 135 (100\%) received noncontract chest CT

- 36 received brain MRI:

- $17(47 \%)$ had brain MRI with and without IV contrast - $7(19 \%)$ received head and neck MRA

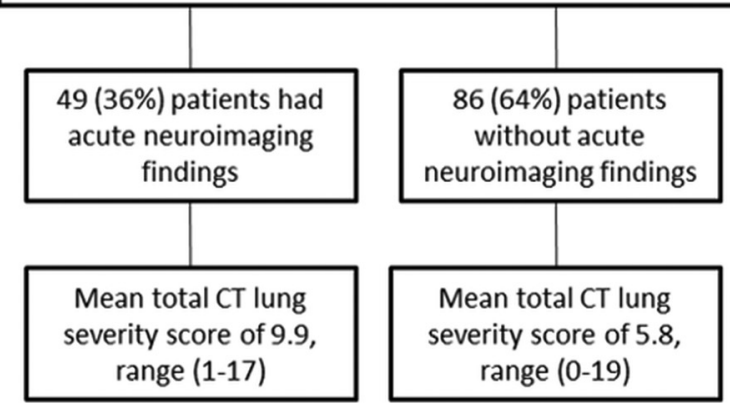

FIG 1. Flow chart of the study.

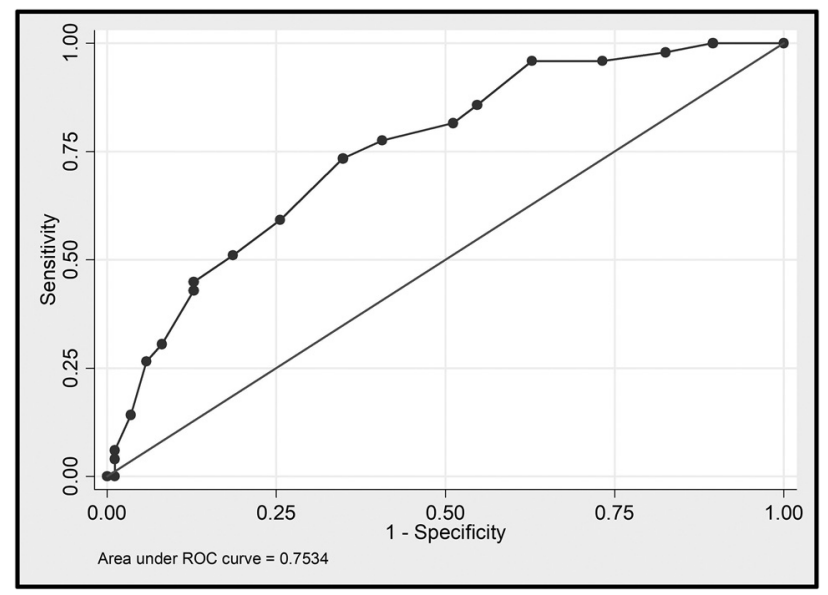

FIG 2. Receiver operating characteristic curve showing the sensitivity and specificity of the CT lung severity score as a predictor of acute neuroimaging abnormalities in patients with COVID-19 with neurologic symptoms. The area under the curve corresponds to the accuracy. $\mathrm{ROC}$ indicates receiver operating characteristic.

Demographics, medical history, and neurologic clinical characteristics are shown in the Online Supplemental Data.

\section{Association between Neuroimaging and CT Chest Findings}

A total of 132 noncontrast brain CT scans, 36 brain MR images, and 135 noncontrast chest CT scans were reviewed. Of these, 7 (19\%) patients underwent head and neck MRA, while 17 (47\%) patients had brain MR imaging with and without IV contrast, of
Excluded: 37 patients without available Chest CT and neuroimaging which only 6 scans included both 3DFLAIR and 3D T1-weighted postcontrast images. Of the 135 patients, 49 (36\%) had acute abnormalities on neuroimaging and $86(64 \%)$ patients had nonacute neuroimaging findings. The patients with acute abnormalities on neuroimaging had a significantly higher mean CT lung severity score versus those without acute neuroimaging abnormalities (9.9 [SD, 4.2] versus 5.8 [SD, 4.3], $P<.001)$. Compared with patients without acute abnormalities on neuroimaging, patients with acute abnormalities on neuroimaging were more likely to present with ischemic stroke (40 [82\%] versus 11 [13\%], $P<.0001)$ and were more likely to have either GGOs or consolidation (46 [94\%] versus 73 [84\%], $P=.01)$. A threshold CT lung severity score of $>8$ was found to be $74 \%$ sensitive and $65 \%$ specific for acute abnormalities on neuroimaging (Fig 2).

None of the remaining neurologic symptoms and patient characteristics, including age and medical history, demonstrated a significant statistical difference. The average time interval between the lung CT and brain imaging was 6 days.

\section{Neuroimaging Characteristics}

The neuroimaging hallmark of these patients was acute ischemic infarcts in 38 (28\%), intracranial hemorrhage in $14(10 \%)$, and white matter disease in 22 (36\%). Of the ischemic infarcts, 21 (15\%) were large, 10 (7\%) were small/watershed infarcts, 5 (4\%) were cardioembolic, and 2 (1\%) were hypoxic-ischemic encephalopathy. Microhemorrhage in 7 (19\%) was the most common intracranial hemorrhage followed by subarachnoid hemorrhage in 4 (3\%). The most frequent MR imaging findings of white matter disease were nonconfluent punctate multifocal T2/FLAIR hyperintense lesions with associated microbleeds in 7 (19\%) (4 [11\%] with and 3 [8\%] without associated restricted diffusion) and confluent symmetric T2/FLAIR hyperintensity involving the deep and subcortical white matter in 4 (11\%) (2 [5\%] with and 2 [5\%]) without associated restricted diffusion) (Fig 3). Neuroimaging characteristics are shown in Table. Patients with nonacute neuroimaging findings had normal scan findings; varying degrees of chronic microvascular ischemic disease, remote infarcts, or chronic demyelinating disease, but no acute abnormalities on neuroimaging. The median time from hospital admission to brain imaging was 9 days. Of the 49 (36\%) patients with acute abnormalities on neuroimaging, 13 were in the intensive care unit and on mechanical ventilation at the time of neuroimaging, with a mean duration of 12 days.

\section{CT Chest Characteristics}

Of the 135 patients, the predominant CT chest patterns were peripheral, either GGOs or consolidation (Online Supplemental 


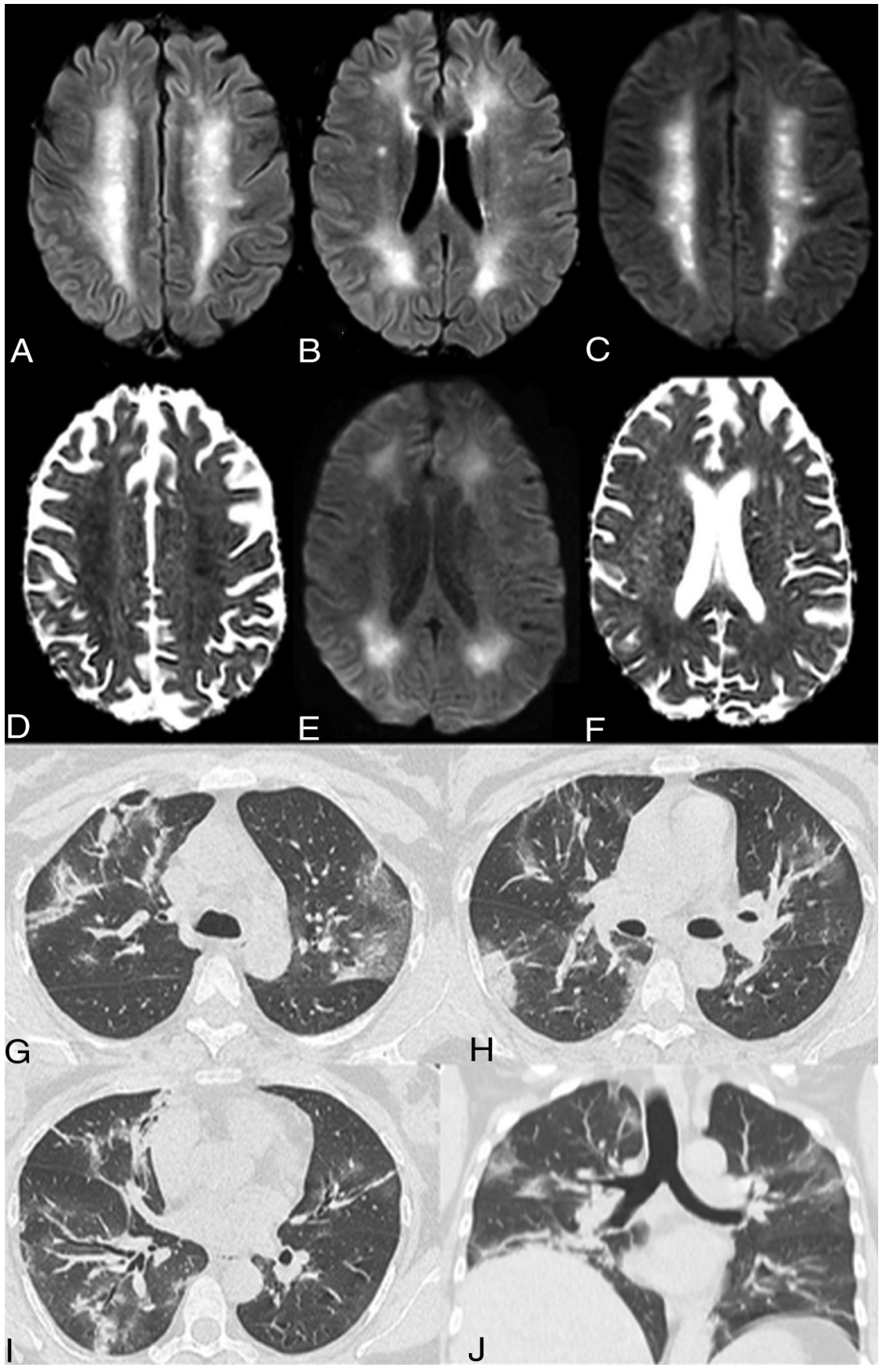

FIG 3. Acute leukoencephalopathy. A 48-year-old man without a history of seizures presented with convulsions and altered mental status. Extensive and confluent symmetric deep and subcortical white matter FLAIR hyperintensities in the bilateral centra semiovale $(A)$ and periventricular frontal and parietal regions $(B)$ with associated mild restricted diffusion on DWV/ADC images, most prominent in the centra semiovale $(C$ and $D)$ and peritrigonal regions $(E$ and $F)$. No associated enhancement or microbleed was seen on the $\Pi$ postcontrast and SWI (not shown). Coronal MPR and axial noncontrast images in lung windows demonstrate mixed ground-glass and consolidative opacities in all lobes with a lower lung and peripheral predominance (G-J). The CT lung severity score was 16 (right upper lobe, right lower lobe, left upper lobe, left lower lobe, 25\%-49\% and right middle lobe $50 \%-75 \%$ ). The chest CT scan was obtained 8 days after the initial onset of respiratory symptoms.

Data). Sixteen (12\%) patients had neither GGOs nor consolidation. Of the 119 (88\%) patients with either GGOs, consolidation, or both, 46 had only GGOs without consolidation, and 7 patients had consolidation without GGOs. The most involved was the left lower lobe in 102 (75\%), followed by the right lower lobe in 97 (72\%). One hundred patients $(81 \%)$ had peripheral predominant distribution, and 61 (45\%) had lower zone involvement. The mean CT lung severity score was 7.3 [SD, 4.1] (range, 0-19).

\section{DISCUSSION}

In this global, multi-institutional study, we found that patients with COVID-19 with neurologic symptoms and acute abnormalities on neuroimaging had, on average, a significantly higher CT lung severity score compared with those without acute neuroimaging findings. A threshold CT lung severity score of $>8$ was found to be $74 \%$ sensitive and $65 \%$ specific for acute abnormalities on neuroimaging. To the best of our knowledge, this is the first study describing the brain and lung imaging correlation in patients with COVID-19. Our findings show that compared with patients without acute abnormalities on neuroimaging, patients with acute abnormalities on neuroimaging were more likely to present with ischemic stroke and were more likely to have either GGOs or consolidation. The neuroimaging features of these patients were variable without a specific pattern but were dominated by acute ischemic infarct, intracranial hemorrhage, and leukoencephalopathy. The predominant CT chest pattern was peripheral GGOs with or without consolidation. We also reported 2 cases of probable COVID-19-associated carotidynia or the new term, Transient Perivascular Inflammation of the Carotid artery (TIPIC) syndrome. ${ }^{18}$

Although accumulating evidence suggests that patients with severe COVID-19 might have a cytokine storm syndrome, resulting in a multiorgan inflammatory response, the underlying pathophysiology of brain and lung association in COVID-19 remains unclear. ${ }^{9,19,20}$ The multisystemic manifestations in COVID-19 infection, including central neurologic symptoms, can be potentially explained by the presence of angiotensin-converting enzyme 2 receptors in multiple human organs, including the brain and lungs. ${ }^{10,21}$ Currently, however, we have a poor mechanistic 


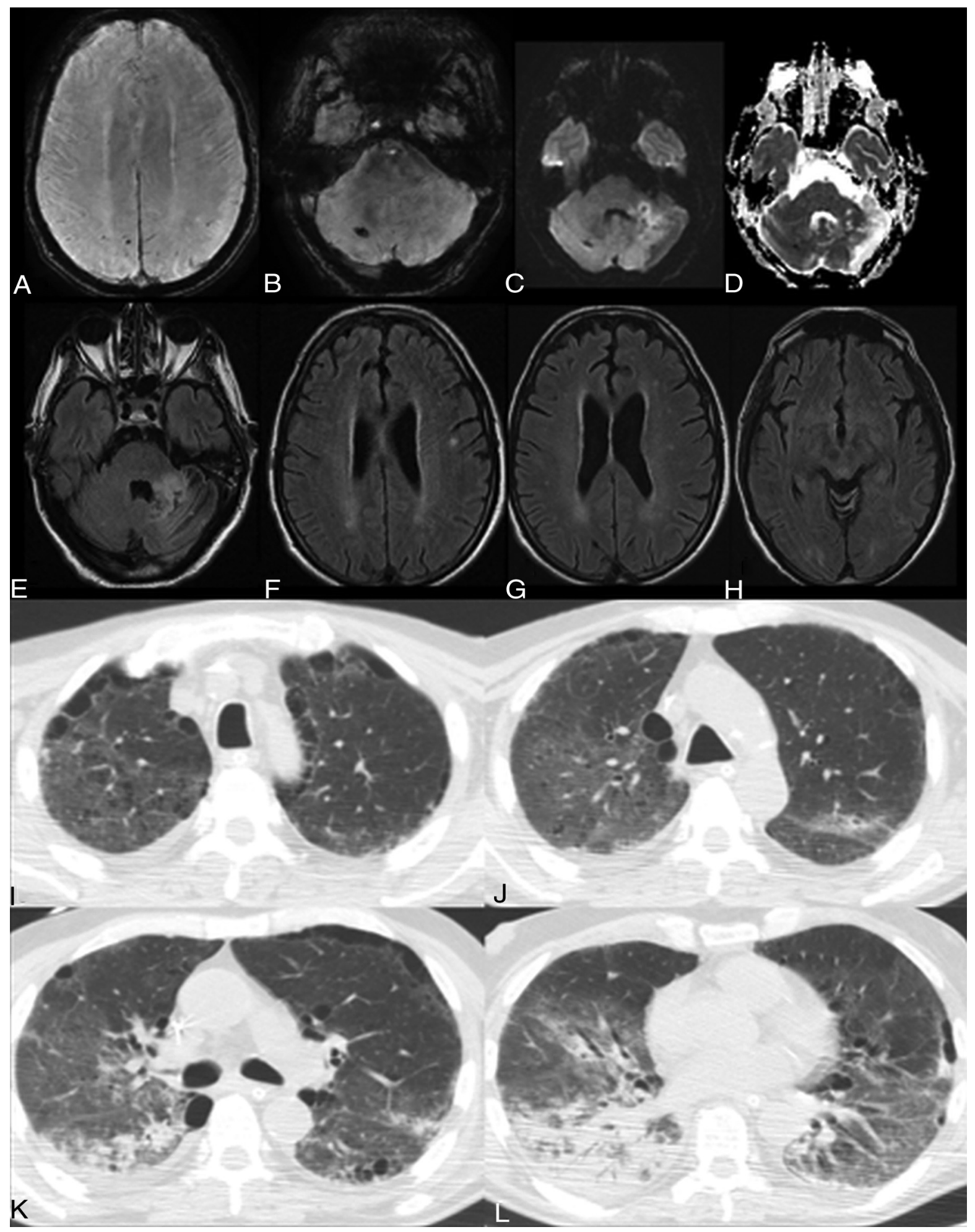

FIG 4. Acute infarct, multifocal microbleeds, and subcortical white matter FLAIR hyperintensity. A 65-year-old man presented with altered mental status and ischemic stroke. Available prior brain MR imaging findings from 1 month ago were normal. $A$ and $B$, New punctate microbleeds on gradient-echo sequences in the right superior parietal lobule, left inferior parietal lobule, and right cerebellum. Note a small area of restricted diffusion in the anterior left cerebellum on DWI/ADC images $(C-D)$ with FLAIR hyperintensities $(E)$, consistent with acute infarct. New punctate deep and subcortical white matter FLAIR hyperintensities without associated hemorrhage or restricted diffusion in the left-greater-than-right frontal $(F)$, parietal $(G)$, and occipital lobes $(H)$. Axial chest CT images show right apical GGOs and biapical paraseptal emphysema (I). Bilateral GGOs are right greater than left (l). Patchy consolidations in the apical segment of the lower lobe of the right lung (K). Extensive consolidation with an air bronchogram in the right lower lobe (L). The CT lung severity score was 14 . The chest CT scan was obtained 10 days after initial onset of respiratory symptoms. 


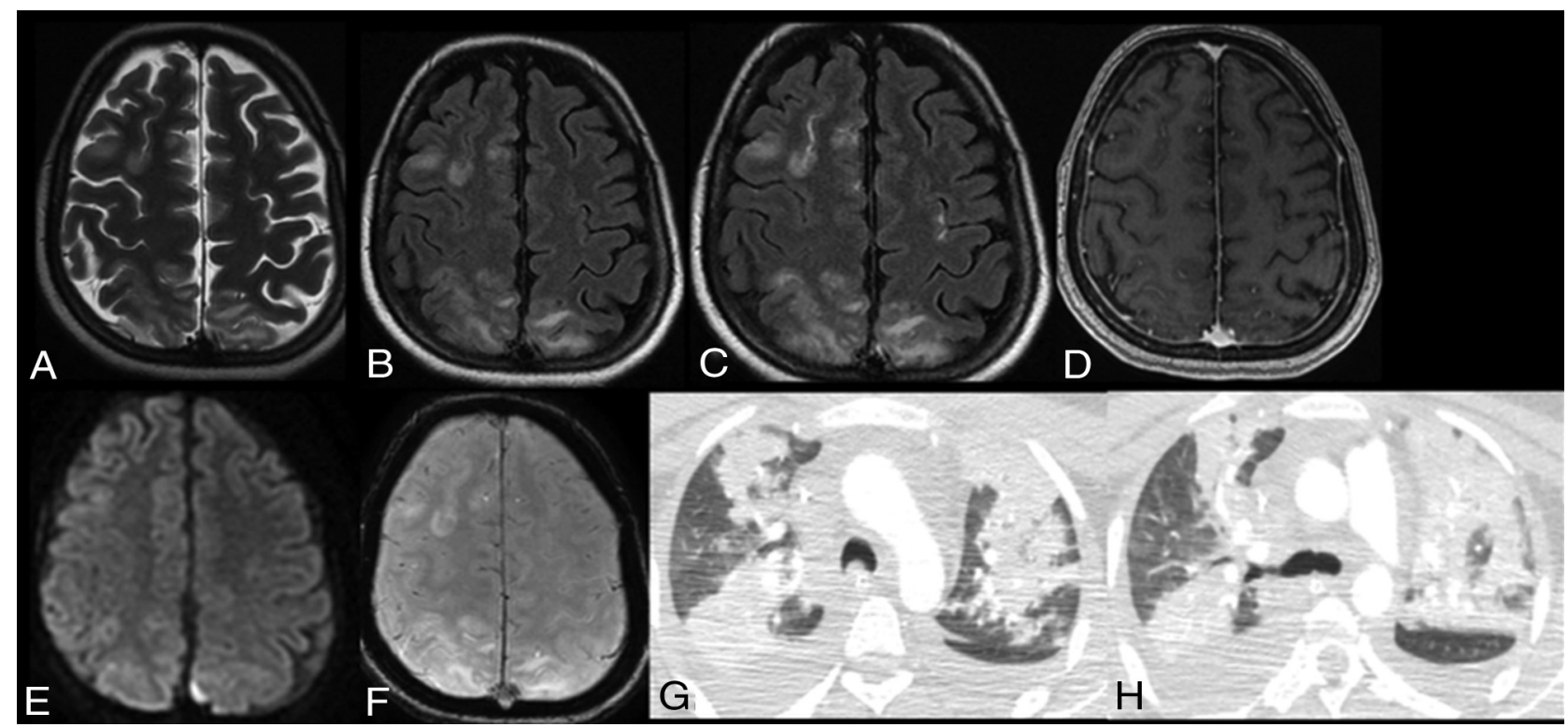

FIG 5. PRES. A 25-year-old woman presented with altered mental status and ischemic stroke. $A$ and $B$, Extensive cortical and subcortical areas of T2/FLAIR hyperintensity in the right frontal and bilateral parietal lobes. Associated patchy and confluent leptomeningeal enhancement is seen only on FLAIR postcontrast image $(C)$ but not on $T 1$ postcontrast image $(D)$. Note no restriction diffusion or hemosiderin on DWI and gradient-echo sequences $(E$ and $F)$. Axial chest $C T$ scans show new extensive bilateral patchy consolidations in the upper lobes with bilateral rightmore-than-left pleural effusions $(G$ and $H$ ). The CT lung severity score was 12 . The chest $C T$ scan was obtained 7 days after the initial onset of respiratory symptoms.

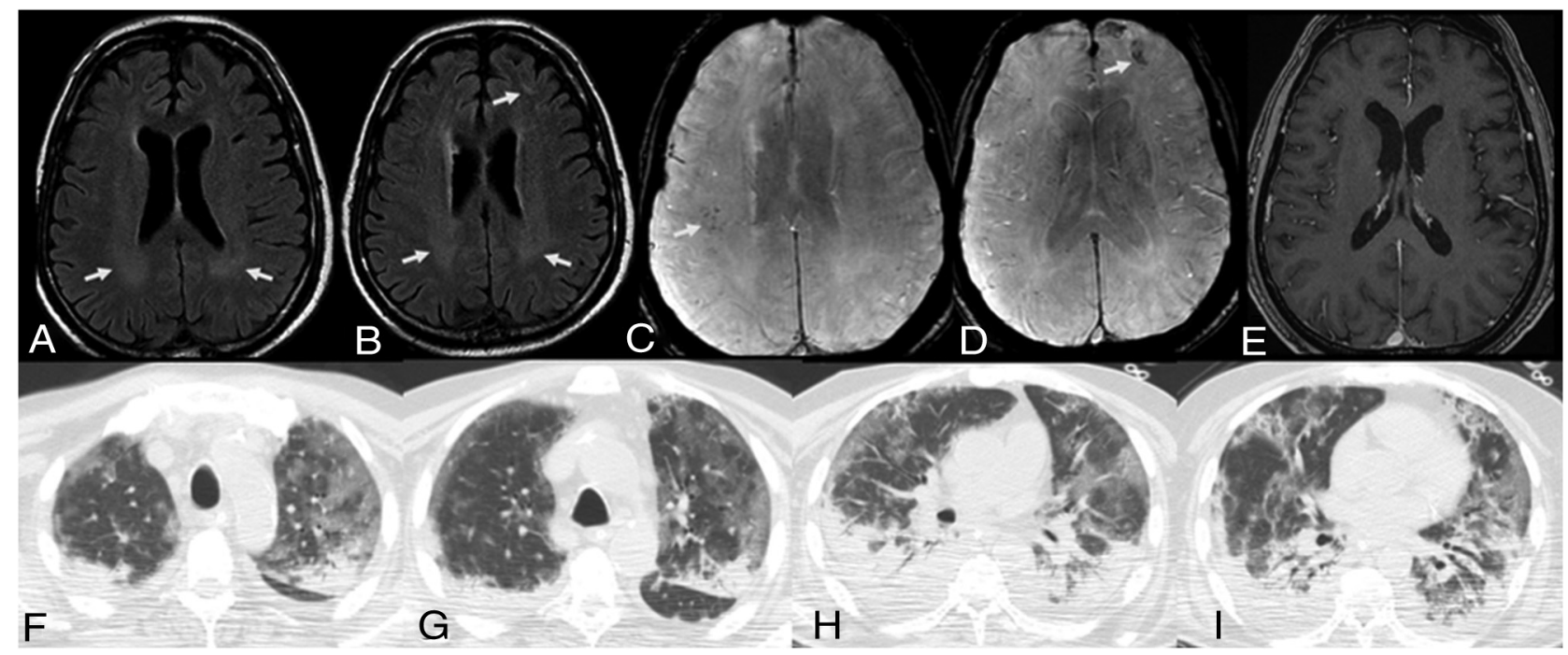

FIG 6. Multifocal microbleeds and deep white matter FLAIR hyperintensities. A 63-year-old man presented with altered mental status. Available prior brain MR imaging findings from 3 months ago were normal. Note confluent periventricular white matter T2/FLAIR hyperintensities, most prominent in the peritrigonal regions $(A$ and $B$ ), with new punctate microbleeds on gradient-echo sequences in the right frontoparietal regions $(C)$ and left frontal lobe $(D)$. No associated enhancement is seen on the T1 postcontrast image. Axial chest CT images in lung windows show bilateral, peripheral, predominant ground-glass opacities and dependent consolidations in the upper and lower lobes ( $F-l)$. Note air bronchograms in both lower lobes ( $H$ and $I)$. The CT lung severity score was 18 . The chest $C T$ scan was obtained 11 days after the initial onset of respiratory symptoms.

understanding of the neurologic manifestations, whether these arise from critical illness or from direct CNS invasion of SARS$\mathrm{CoV}-2$ or represent treatment-related complications from current COVID-19 therapies including intensive care unit-related complications. ${ }^{9,22}$ PRES and multifocal cerebral thrombotic microangiopathy were reported to be complications of the new COVID-19 antiviral and immunosuppressive drugs such as tocilizumab, which was used in 12 patients in our series. ${ }^{22,23}$

Postmortem neuropathologic examinations in 18 patients with COVID-19 showed only hypoxic changes without signs of encephalitis. ${ }^{24,25}$ Furthermore, it has been suggested that recruitment of immune cells by either direct viral infection or immune- 


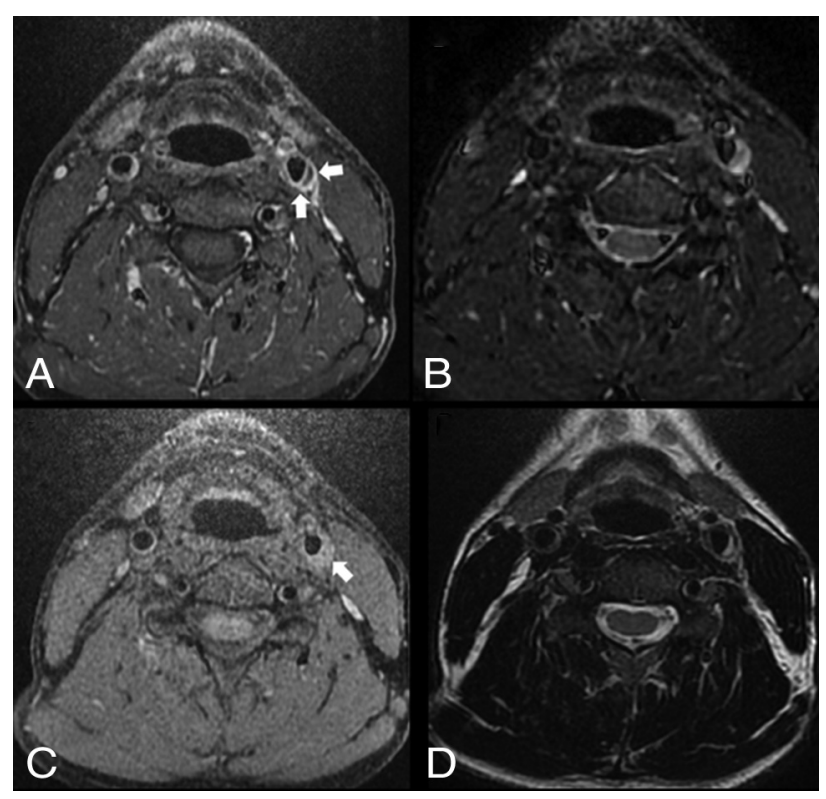

FIG 7. Left TIPIC (carotidynia). A 40-year-old man without significant medical history or known trauma presented with myoclonus and acute tenderness overlying the left carotid artery with increased pulsation. Axial $\Pi$ fat-saturated postcontrast image shows asymmetric enhancement and nonstenotic thickening of the left common carotid wall ( $A$, arrows), with $\Pi$ precontrast hyperintensity of a crescent-shaped subintimal focus ( $C$, arrow), which is also hyperintense on $T 2$ fat-saturated and T2-weighted ( $B$ and $D$ ) images. Differential considerations include carotidynia or dissection. However, given the significantly improved symptoms after anti-inflammatory treatment, the lack of luminal stenosis, and the patient's neck pain characteristics, findings are most consistent with carotidynia. No follow-up imaging was available.

mediated damage across different organ vascular beds may lead to endothelial damage, which results in stroke, hemorrhage, and thrombosis. ${ }^{9,26}$ Additionally, it is thought that this latter triggers extensive pulmonary intravascular immunothrombosis and potentially also promotes intraplaque inflammation and destabilization. ${ }^{27}$ This could be the underlying mechanism in our 2 patients with COVID-19-associated TIPIC. Although the differential diagnosis may include dissection, the combination of clinical and imaging criteria was most consistent with carotidynia. These criteria include the presence of acute pain overlying the carotid artery with increased pulsation, significantly improved symptoms after anti-inflammatory treatment, no significant medical history or known trauma, eccentric perivascular wall thickening, and enhancement without significant luminal stenosis. ${ }^{18}$

Our findings concur with additional published literature including Mao et $\mathrm{al}^{10}$ from Wuhan, China, who reported that 27 (51\%) of the patients with CNS symptoms had severe respiratory disease, but there was no description of brain and lung imaging features. Similar to other investigators who described neuroimaging features in severe COVID-19, $7,8,13$ our findings showed multiple patterns of white matter disease and hemorrhage; however, none of these studies have demonstrated an association of neuroimaging with the CT lung severity score or with a specific CT lung pattern. Consistent with previous studies, ${ }^{7,8,13}$ confluent white matter T2 hyperintensities with or without restricted diffusion reported in 2 of our patients (Fig 3) are nonspecific but can be related to delayed posthypoxic leukoencephalopathy; however, the main differential diagnoses include hypoxic-ischemic injury, infectious or autoimmune encephalitis, hypoglycemia, and seizure. Our chest CT findings largely concur with previous investigations insofar as the chest CT hallmark of COVID-19 infection is ground-glass and consolidative pulmonary opacities, with bilateral and peripheral lung distribution. ${ }^{2-5}$

Indeed, there is minimal research in the area where acute lung injury and severe brain injury coincide. Acute lung injury occurs in $20 \%$ of patients with brain injury and is associated with a poor outcome. ${ }^{14}$ Patients with acute respiratory distress syndrome are at risk of brain injury through hypoxemia and/or proinflammatory mediators that connect both the brain and the lungs. ${ }^{15-17}$ It is hypothesized that sensory neurons in the airways may provide a sensing mechanism to detect the inflammatory intensity in the lung, creating a feedback mechanism to the brain, thus illustrating lung-brain communication. ${ }^{15}$ Hippocampal neuronal cell damage was demonstrated in a porcine acute lung injury model with increased serum proinflammatory mediators. ${ }^{17}$ Nevertheless, the underlying pathophysiology of brain-lung association in patients with COVID-19 remains unclear. The identification of the correlation between lung and brain findings could be important for targeting optimized therapies, particularly in stroke prevention. Our findings could be explored in future studies to potentially develop preventive and therapeutic strategies that can improve outcomes in patients with COVID-19.

There are several important limitations to our study including a retrospective design. Second, the known temporal variation of chest CT findings may affect the uniformity of the CT lung severity score; ${ }^{4,28}$ however, only scans with the greatest severity were selected, and all were performed approximately 7-12 days after the initial onset of respiratory symptoms, to minimize this limitation. Third, the impaired consciousness of the patients limited the ability to perform clinicoradiologic correlations, particularly in assessing disease severity and clinical outcomes. Fourth, patients with unstable conditions with critical illness may have delayed or no imaging, which may affect accurate capture of imaging characteristics and therefore underestimate their prevalence. Fifth, compared with MR imaging, CT has a low sensitivity for white matter disease, which may also underestimate the results in patients who only underwent CT neuroimaging. Finally, it is difficult to establish the coincidence of imaging findings versus causality in critically ill patients and to differentiate the imaging findings from those of current COVID-19 therapyrelated complications.

\section{CONCLUSIONS}

Our study demonstrates that patients with COVID-19 with neurologic symptoms and acute abnormalities on neuroimaging had a higher CT lung severity score compared with patients with COVID-19 with neurologic symptoms and nonacute neuroimaging findings. Future larger datasets with imaging-pathologic correlation may help in understanding the common mechanisms of brain and lung injury. 


\begin{tabular}{|c|c|}
\hline Neuroimaging Characteristics & All Patients $(n=135), \mathrm{CT}(n=132)$ or MR imaging $(n=36)$ \\
\hline \multicolumn{2}{|l|}{ T2/FLAIR white matter signal abnormality } \\
\hline $\begin{array}{l}\text { Nonconfluent punctate deep and subcortical white matter } \\
\text { disease }\end{array}$ & $22 / 36(61)$ \\
\hline Isolated, nonspecific & $9 / 36(25)$ \\
\hline Associated restricted diffusion only ${ }^{b}$ & $6 / 36(17)$ \\
\hline Associated microhemorrhage only & $3 / 36(8)$ \\
\hline Associated microhemorrhage and restricted diffusion ${ }^{\mathrm{b}}$ & $4 / 36(11)$ \\
\hline $\begin{array}{l}\text { Confluent symmetric } \mathrm{T} 2 \text { hyperintensity without restricted diffusion } \\
\text { or hemorrhage }\end{array}$ & $2 / 36(5)$ \\
\hline $\begin{array}{l}\text { Confluent symmetric } T 2 \text { hyperintensity with mild restricted } \\
\text { diffusionc }\end{array}$ & $2 / 36(5)$ \\
\hline Enhancement (MR imaging with and without IV contrast) & $2 / 17(12)$ \\
\hline Leptomeningeal $^{\mathrm{d}}$ & $2 / 17(12)$ \\
\hline Parenchymal $^{\mathrm{e}}$ & $0 / 17(0)$ \\
\hline \multicolumn{2}{|l|}{ Cranial nerves } \\
\hline Acute ischemic infarcts & $36 / 135(27)$ \\
\hline Vascular territory & $21 / 135(15)$ \\
\hline Small/watershed infarcts & $10 / 135(7)$ \\
\hline Cardioembolic & $5 / 135(4)$ \\
\hline Intracranial hemorrhages & $14 / 135(10)$ \\
\hline Parenchymal & $3 / 135(2)$ \\
\hline Subarachnoid & $4 / 135(3)$ \\
\hline Microhemorrhage & $7 / 36(19)$ \\
\hline Acute leukoencephalopathy ${ }^{c}$ & $4 / 36(11)$ \\
\hline PRES & $3 / 36(8)$ \\
\hline Hypoxic-ischemic encephalopathy & $2 / 36(5)$ \\
\hline TIPIC $^{f}$ & $2 / 7(28)$ \\
\hline \multirow{2}{*}{\multicolumn{2}{|c|}{$\begin{array}{l}\text { a Numbers in parentheses are percentages. } \\
\text { bThought to be most consistent with acute lacunar infarcts with a few associated microhemorrhages (Figs } 4 \text { and 6). }\end{array}$}} \\
\hline & \\
\hline \multicolumn{2}{|c|}{ ‘Acute leukoencephalopathy. A 48-year-old man without a history of seizures presented with convulsion and altered mental status (Fig 3). } \\
\hline \multicolumn{2}{|c|}{ dSeen on FLAIR postcontrast only and likely related to PRES (Fig 5). } \\
\hline \multicolumn{2}{|c|}{$\begin{array}{l}\text { eSeptic emboli with atypical left parietal abscess. A 70-year-old woman with high blood pressure, chronic kidney disease, and type } 2 \text { diabetes mellitus. Long admission in } \\
\text { the intensive care unit with intubation for COVID-19 and bilateral pneumonia. She presented with alteration of mental state and difficulty to progress in the weaning pro- } \\
\text { cess. No history of malignancy (Online Supplemental Data). }\end{array}$} \\
\hline Left TIPIC (carotidynia). A 40 -year-old man without significant medical history or $k$ & a presented with myoclonus and acute tenderness overlying the left ca- \\
\hline
\end{tabular}

\section{ACKNOWLEDGMENTS}

We thank all patients and their families involved in the study.

Disclosures: Ana Ramos-UNRELATED: Employment: Hospital 12 de Octubre. Nuria Bargalló - UNRELATED: Employment: Domicilio. Soma Sengupta—UNRELATED: Consultancy: NovoCure; Grants/Grants Pending: R01, R21; Payment for Lectures Including Service on Speakers Bureaus: University of Kentucky, Neurology Grand Rounds; Patents (Planned, Pending or Issued): patents but no money involved; Other: equity in a start-up, currently no finances in the start-up. Achala VagalRELATED: Grant: National Institutes of Health, Comments: R01 NIH/NINDS NS103824, RF1 NIH/NINDS/NIA NS117643, R01 NIH/NINDSNS100417, NIH/NINDS IU01NS100699, NIH/NINDS U01NS110772.* *Money paid to the institution.

\section{REFERENCES}

1. Novel coronavirus (2019-nCoV) situation reports. https://www. who.int/emergencies/diseases/novel-coronavirus-2019/situationreports. Accessed April 4, 2020

2. Bernheim $A$, Mei $X$, Huang $M$, et al. Chest $C T$ findings in coronavirus disease-19 (COVID-19): relationship to duration of infection. Radiology 2020;295:200463 CrossRef Medline

3. Chung M, Bernheim A, Mei X, et al. CT imaging features of 2019 novel coronavirus (2019-nCoV). Radiology 2020;295:202-07 CrossRef Medline

4. Pan F, Ye T, Sun P, et al. Time course of lung changes at chest CT during recovery from coronavirus disease 2019 (COVID-19). Radiology 2020;295:715-21 CrossRef Medline

5. Ng MY, Lee EY, Yang J, et al. Imaging profile of the COVID-19 infection: radiologic findings and literature review. Radiology: Cardiothoracic Imaging 2020;2:e200034 CrossRef
6. Mahammedi A, Saba L, Vagal A, et al. Imaging in neurological disease of hospitalized patients with COVID-19: an Italian multicenter retrospective observational study. Radiology 2020;297:E270-73 CrossRef Medline

7. Radmanesh A, Derman A, Lui YW, et al. COVID-19-associated diffuse leukoencephalopathy and microhemorrhages. Radiology 2020;297:E223-27 CrossRef Medline

8. Kremer S, Lersy F, de Sèze J, et al. Brain MRI findings in severe COVID-19: a retrospective observational study. Radiology 2020;297: E242-51 CrossRef Medline

9. Chougar L, Shor N, Weiss N, et al. CoCo Neurosciences Study Group. Retrospective observational study of brain magnetic resonance imaging findings in patients with acute SARS-CoV-2 infection and neurological manifestations. Radiology 2020;297:E313-23 CrossRef Medline

10. Mao L, Jin H, Wang M, et al. Neurologic manifestations of hospitalized patients with coronavirus disease 2019 in Wuhan, China. JAMA Neurol 2020;77:683-90 CrossRef Medline

11. Li Y, Li M, Wang M, et al. Acute cerebrovascular disease following COVID-19: a single center, retrospective, observational study. Stroke Vasc Neurol 2020;5:279-84 CrossRef Medline

12. Klironomos S, Tzortzakakis A, Kits A, et al. Nervous system involvement in COVID-19: results from a retrospective consecutive neuroimaging cohort. Radiology 2020;297:E324-34 CrossRef Medline

13. Kandemirli SG, Dogan L, Sarikaya ZT, et al. Brain MRI findings in patients in the intensive care unit with COVID-19 infection. Radiology 2020;297:E232-35 CrossRef Medline

14. Mascia L. Acute lung injury in patients with severe brain injury: a double hit model. Neurocrit Care 2009;11:417-26 CrossRef Medline 
15. Moldoveanu B, Otmishi $\mathrm{P}$, Jani $\mathrm{P}$, et al. Inflammatory mechanisms in the lung. I Inflamm Res 2009;2:1-11 Medline

16. Tracey KJ. The inflammatory reflex. Nature 2002;420:853-59 CrossRef Medline

17. Raabe A, Wissing $\mathrm{H}$, Zwissler B. Brain cell damage and S-100B increase after acute lung injury. Anesthesiology 2005;102:713-14 CrossRef Medline

18. Lecler A, Obadia M, Savatovsky J, et al. TIPIC syndrome: beyond the myth of carotidynia, a new distinct unclassified entity. AJNR Am J Neuroradiol 2017;38:1391-98 CrossRef Medline

19. Mehta P, McAuley DF, Brown M, et al. COVID-19: consider cytokine storm syndromes and immunosuppression. Lancet 2020;395:103334 CrossRef Medline

20. Grau AJ, Buggle F, Becher H, et al. Recent bacterial and viral infection is a risk factor for cerebrovascular ischemia: clinical and biochemical studies. Neurology 1998;50:196-203 CrossRef Medline

21. Hamming I, Timens W, Bulthuis MLC, et al. Tissue distribution of ACE2 protein, the functional receptor for SARS coronavirus: a first step in understanding SARS pathogenesis. J Pathol 2004;203:631-37 CrossRef Medline
22. Zubair AS, McAlpine LS, Gardin T, et al. Neuropathogenesis and neurologic manifestations of the coronaviruses in the age of coronavirus disease 2019: a review. JAMA Neurol 2020;77:1018-27 CrossRef Medline

23. Tocilizumab-associated multifocal cerebral thrombotic microangiopathy. Neurology Clinical Practice. January 29, 2016. https:// cp.neurology.org/content/6/3/e24.short. Accessed September 7, 2020

24. Solomon IH, Normandin E, Bhattacharyya S, et al. Neuropathological features of Covid-19. N Engl J Med 2020;383:989-92 CrossRef Medline

25. Matschke J, Lütgehetmann M, Hagel C, et al. Neuropathology of patients with COVID-19 in Germany: a post-mortem case series. Lancet Neurol 2020;19:919-29 CrossRef Medline

26. Varga Z, Flammer AJ, Steiger P, et al. Endothelial cell infection and endotheliitis in COVID-19. Lancet 2020;395:1417-18 CrossRef Medline

27. McGonagle D, O'Donnell JS, Sharif K, et al. Immune mechanisms of pulmonary intravascular coagulopathy in COVID-19 pneumonia. Lancet Rheumatol 2020;2:e437-45 CrossRef Medline

28. Wang Y, Dong C, Hu Y, et al. Temporal changes of CT findings in 90 patients with COVID-19 pneumonia: a longitudinal study. Radiology 2020;296:E55-64 CrossRef Medline 